\title{
La satisfacción estudiantil universitaria: análisis estratégico a partir del análisis de factores
}

\section{RESUMEN}

Este estudio tiene como objetivo presentar estrategias para lograr la satisfacción estudiantil partiendo de su medición y del análisis factorial del constructo en una universidad mexicana, usando un instrumento de medición de satisfacción estudiantil universitaria (SEUing). Trata de un estudio exploratorio basado en una revisión bibliográfica del tema. Mediante un muestreo no probabilístico fueron recolectados 100 datos en la carrera de Ingeniería Industrial, a partir de los cuales, previa demostración de la adecuación muestral (determinante de la matriz de correlación $=8,93 \times 10^{-6} \mathrm{y}$ el estadístico KMO fue de 0.849 ), se realizó un análisis de factores. Usando una rotación Varimax, se identificaron 4 dimensiones y fueron denominadas: Enseñanza, Organización Académica, Vida Universitaria, Infraestructura y Servicios Universitarios; con consistencias internas de $a_{1}=0,894 ; a_{2}=0,869 ; a_{3}=0,822$; $a_{4}=0,745$, respectivamente. Explicando un $67 \%$ de la varianza total. Las pruebas estadísticas realizadas (significativas al $5 \%$ ), el análisis de fiabilidad (alfa de Cronbach $>0,70$ ), evidencian la validez y fiabilidad de la escala SEU usada.

Palabras clave: análisis de factores, satisfacción estudiantil, universidad

A College student satisfaction: Strategic ANALYSIS FROM FACTOR ANALYSIS

\section{ABSTRACT}

This study aims to present strategies for student satisfaction based on measurement and factorial analysis of this construct ina Mexican University, using a measuring college student satisfaction (SEUing ). This is an exploratory study based on a review of the literature. Using a non-probability sampling 100 data were collected in Industrial Engineering, from which, upon proof of sampling adequacy (determinant of correlation matrix $=8.93 \times 10-6$ and $\mathrm{KMO}$ statistic was 0.849 ), factor analysis was performed. Using a Varimax rotation, four dimensions were identified and were named : Teaching, Academic Organization, Campus Life, Facilities and University Services, with Internal Consistencies of $a_{1}=$ $0.894 ; a_{2}=0.869 ; a_{3}=0.822 ; a_{4}=0.745$, respectively. Explaining $67 \%$ of the total variance, Statistical tests performed (significant at $5 \%$ ) , the analysis of reliability (Cronbach's alpha $>0.70$ ), demonstrate the validity and reliability of the scale used SEU.

Keywords: factor analysis, student satisfaction, university

\section{INTRODUCCIÓN}

La gestión de la calidad, sin duda alguna, se ha convertido en un medio catalizador de las buenas prácticas gerenciales en las organizaciones a nivel mundial. Además de las funciones inherentes a su misión, las empresas tienen un rol importante en el impulso de los esfuerzos de mejora de la calidad en la educación superior mediante la transferencia de conocimiento, y la experiencia de los procesos de calidad y las prácticas para implantarlos (Evans y Lindsay, 2008).

Uno de los principios universales de la gestión de la calidad es el enfoque al cliente. Al respecto, la Organización Internacional para la Normalización (ISO) establece que, las organizaciones dependen de sus clientes y por lo tanto deberían comprender sus necesidades actuales y futuras, satisfacer los requisitos y esforzarse en exceder sus expectativas (ISO, 2005). Además, la ISO 9001 (ISO, 2008), establece como una de las medidas del desempeño del sistema de gestión de la calidad que la organización debe realizar el seguimiento de la información relativa a la percepción del cliente con respecto al cumplimiento de sus requisitos por parte de la organización, así mismo que deben determinarse los métodos para obtener y utilizar dicha información. Las instituciones educativas, en esa búsqueda de oportunidades para mejorar, han venido identificando modelos para evaluar la satisfacción estudiantil en sintonía con las tendencias en gestión de la calidad y excelencia en el desempeño. Como lo señalan Álvarez y Vernaza (2013), lograr conocer la dimensión de la satisfacción de los estudiantes con la institución a la cual concurren, permitirá identificar aspectos tanto positivos como negativos, siendo estos últimos fundamentales al momento de determinar estrategias de mejora de la educación. Por su parte, Alves y Raposo (2004), plantean la importancia de encontrar formas fiables de medir la satisfacción estudiantil universitaria, ya que permitiría a las instituciones conocer su realidad, compararla con la de los otros competidores, y analizarla a lo largo del tiempo

Entre los modelos para medir la satisfacción estudiantil en el contexto universitario se destacan: Gento y Vivas (2003) quienes elaboran un instrumento para conocer la satisfacción de los estudiantes por sus resultados y experiencias en

* Dpto. de Ingeniería Industrial, Universidad de Sonora, México. E-mail: mcadena@guaymas.uson.mx ** Universidad de Carabobo, Venezuela. E-mail: aamejiasa@gmail.com

*** Dpto. de Ingeniería Industrial, Universidad de Sonora, México. E-mail: avega@caborca.uson.mx ****Dpto. de Ingeniería Industrial, Universidad de Sonora, México. E-mail: jovaqui@caborca.uson.mx 
Venezuela; Alves y Raposo (2004), quienes miden la satisfacción estudiantil en una universidad de Portugal a partir de índices; el SEUing de Martínez y Mejías (2009), quienes proponen un instrumento para su medición; Torres y Sepulveda (2009) evalúan la satisfacción de estudiantes chilenos mediante el Análisis de Factores; de la Fuente, Marzo y Reyes (2010) quienes desarrollan y evalúan una escala de medida de satisfacción de los alumnos de una Universidad Chilena; Vergara y Quesada (2011), quienes analizan la satisfacción de los estudiantes en una Universidad Colombiana; Blázquez et al. (2013), quienes identifican los principales indicadores de satisfacción estudiantil en una Universidad Española; entre otros. El modelo presentado por Martínez y Mejías (2009) permite partir de una estructura base que cuenta con 52 variables agrupadas en cuatro dimensiones, el cual se puede ir ajustando de acuerdo con el caso a aplicar. Además de la validación realizada por los autores en una Universidad Venezolana, Candelas et al. (2013), aplican el modelo en el contexto mexicano. Otros investigadores han aplicado el modelo para fines de gestión universitaria.

El objetivo del presente trabajo es realizar un análisis estratégico de la gestión universitaria partiendo de un análisis de los factores que inciden en las percepciones del grado de satisfacción estudiantil de los educandos en una Universidad Mexicana. Para la recopilación de los datos se aplicó el instrumento SEUing, Para la identificación de los factores que determinan la satisfacción estudiantil se utilizó el Análisis de Factores, y para el Análisis Estratégico se desarrolló una matriz DOFA (Debilidades-Oportunidades-FortalezasAmenazas).

Una vez presentada la introducción a manera de estado del arte, se presenta la metodología seguida para el desarrollo de la investigación, básicamente girando en torno al Análisis de Factores y en la validación de la encuesta usada; luego, se presentan y se discuten los resultados, para presentar finalmente, las conclusiones y las referencias.

\section{METODOLOGÍA}

La investigación desarrollada es de tipo descriptiva no experimental. Se seleccionó el modelo de encuesta SEUing propuesto por Martínez y Mejías (2009), el cual presentaba mayor similitud con el caso en estudio. A objeto de conceptualización de la variable, se adopta la definición de Satisfacción Estudiantil planteada por Martínez y Mejías (2009): "nivel del estado de ánimo que poseen los estudiantes con respecto a su institución, como resultado de la percepción que poseen con respecto al cumplimiento de sus necesidades, expectativas y requisitos", o aún más, se puede definir también como "la confirmación o disconfirmación de expectativas previas".

El cuestionario SEUing base seleccionado consta de cincuenta y dos (52) variables agrupadas en cuatro (04) dimensiones, el cual fue aplicado a cien (100) estudiantes en la Universidad de Sonora, en México. A los estudiantes se les dieron instrucciones, explicándoles los objetivos de la aplicación de la encuesta. Una vez, obtenidos los datos, los mismos fueron registrados en una base de datos; luego se aplicó el Análisis de Factores.

El Análisis de Factores tiene como objeto simplificar las múltiples y complejas relaciones que puedan existir entre un conjunto de variables observadas $\mathrm{X}_{1}, \mathrm{X}_{2}, \ldots, \mathrm{X}_{\mathrm{p}}$, tratando de encontrar dimensiones o factores comunes que ligan a las aparentes no relacionadas variables. Concretamente, se trata de encontrar un conjunto de $k<p$ no directamente observables $F_{1}, \quad F_{2}, \ldots, \quad F_{k}$ que expliquen suficientemente a las variables observadas $\left(X_{1}\right.$, $\mathrm{X}_{2}, \ldots, \mathrm{X}_{\mathrm{p}}$ ) perdiendo el mínimo de información, de modo que sean fácilmente interpretables <Principio de Interpretabilidad> y que sean los menos posibles, es decir, k pequeño <Principio de Parsimonia> (Pérez, 2004). Una buena solución factorial es aquella que es sencilla e interpretable (Hair et al. 2010).

Para el análisis estratégico, se usó la matriz DOFA, la cual es una herramienta con la que se generan estrategias luego de hacer un análisis cruzado entre los factores del ambiente externo (Amenazas y Oportunidades) y los factores del ambiente interno <Fortalezas y Debilidades> (Aceves, 2004; Garrido, 2004; Cadena et al. 2013).

\section{RESULTADOS Y DISCUSIÓN}

\subsection{Análisis de Factores}

Como ya se ha señalado, el Análisis de Factores tiene por objeto explicar un conjunto de variables observadas por un pequeño número de variables latentes, o no observadas (Peña, 2002). Esta técnica estudia la estructura en las relaciones entre un grupo de variables como para reducir su número; asume que las correlaciones entre variables no son aleatorias, sino que se deben a la existencia subyacente de factores comunes entre ellas (Garmendia, 2007). Al momento de que el investigador tiene la necesidad de conocer 
las relaciones entre las variables para inferir en resultados como producto de una combinación de las mismas, surge la necesidad de efectuar un Análisis de Factores (Cadena, 2013).

La primera fase del Análisis de Factores es el cálculo de una matriz capaz de expresar la variabilidad conjunta de todas las variables (Pardo y Ruíz, 2002). Para facilitar el análisis de esta matriz, se usan los indicadores KMO y el determinante de la matriz. EI KMO (Kaiser-MayerOlkin) es un coeficiente de correlación parcial que mide la correlación existente entre variables una vez que se han descontado los efectos lineales de otras variables, en caso de que exista adecuación de los datos a un modelo de análisis de factores, esta medida estará próxima a 1 (Vicente y Oliva y Manera, 2003); el determinante de la matriz es un indicador del grado de intercorrelaciones, si este es muy bajo las correlaciones entre las variables son muy altas (Vicente y Oliva y Manera, 2003). Los resultados reportados de $\mathrm{KMO}=0,849$ y

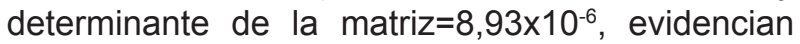
la adecuación muestral para realizar el Análisis de Factores.

A partir de la depuración de los datos, tomando en cuenta los criterios de parsimonia e Interpretabilidad de Thurstone (1947), citado por Pérez, (2004) y Hair et al. (2010), entre otros, la cual establece que se deberán tomar la explicación más simple de aquellas que sean coherentes con los datos, en la tabla 1 se presentan los resultados de la matriz de componentes rotados. Esta matriz fue obtenida utilizando como método de rotación el criterioVarimax (Káiser, 1958), a partir del uso del Software SPSS 20 para hacer las "corridas" de datos.

\subsection{Análisis de Fiabilidad}

De acuerdo con (Prat y Doval, 2003), el coeficiente Alfa de Cronbach indica la consistencia interna del instrumento, tomando valores entre 0 y 1 , siendo cercanos a cero como no existe consistencia interna y cercana a 1 existe consistencia interna. Esta se realiza para evaluar la capacidad del instrumento para medir lo que se desea, permitiendo ser utilizado en diferentes oportunidades y obtener resultados constantes en similares situaciones; el mismo arrojó resultados superiores a 0.700 para las dimensiones identificadas $\left(\alpha_{1}=0,894\right.$; $\left.\alpha_{2}=0,869 ; \alpha_{3}=0,822 ; \alpha_{4}=0,745\right)$. Se considera que el instrumento presenta consistencia interna, por lo que existe homogeneidad de las respuestas de los ítems, esto señala que la escala es fiable para ser utilizado.
Tabla 1. Matriz de componentes rotados

\begin{tabular}{|c|c|c|c|c|}
\hline \multirow{2}{*}{} & \multicolumn{5}{|c|}{ Componentes } \\
\hline & $\mathbf{1}$ & $\mathbf{2}$ & $\mathbf{3}$ & $\mathbf{4}$ \\
\hline V01 &, 858 & & & \\
\hline V19 &, 778 & & & \\
\hline V02 &, 759 & & & \\
\hline V16 &, 718 & & & \\
\hline V04 &, 692 & & & \\
\hline V25 & &, 812 & & \\
\hline V24 & &, 792 & & \\
\hline V23 & &, 716 & & \\
\hline V27 & &, 682 & & \\
\hline V38 & &, 584 & & \\
\hline V43 & & &, 767 & \\
\hline V37 & & &, 713 & \\
\hline V41 & & &, 699 & \\
\hline V40 & & &, 632 & \\
\hline V42 & & &, 541 & \\
\hline V48 & & & &, 766 \\
\hline V47 & & & &, 675 \\
\hline V30 & & & &, 674 \\
\hline V32 & & & &, 652 \\
\hline V45 & & & &, 633 \\
\hline
\end{tabular}

\subsection{Validez del Instrumento}

La validez es un concepto del cual pueden tenerse diferentes tipos de evidencia (Streiner y Normand, 2008; Wiersman y Jurs, 2008; y Babbie, 2009): 1) evidencia relacionada con el contenido, 2) evidencia relacionada con el criterio y 3) evidencia relacionada con el constructo. La validez hace referencia a la capacidad que posee el instrumento de medir la situación en estudio con propiedad. Siendo validado el instrumento a través de los resultados, no por el instrumento por si solo o por la técnica a través de la cual se aplicó (Rodríguez, Ochoa y Pineda, 2012).

Martin (2004), plantea la validez, como el grado en que un instrumento de medida mide aquello que realmente pretende medir o sirve para el propósito para el que ha sido construido; aunque se hablan de diferentes tipos de validez, este es un proceso unitario que permitirá realizar las inferencias e interpretaciones de acuerdo a las puntuaciones obtenidas de la aplicación del instrumento. Considerando ello se presentan los siguientes enfoques para obtener la validez en el presente estudio: 
Tabla 2. Factores que determinan la satisfacción estudiantil Universitaria

\begin{tabular}{|c|l|}
\hline 1 & Mis profesores demuestran dominio acerca de la materia que imparten \\
\hline 19 & Me encuentro satisfecho con el liderazgo de los profesores \\
\hline 2 & Mis profesores me están preparando para comportarme con autonomía e iniciativa \\
\hline 16 & Mis profesores me enseñan a trabajar en equipo \\
\hline 4 & Mis profesores me están capacitando para expresarme en público con seguridad y lenguaje apropiado \\
\hline 25 & La Dirección de la Institución se preocupa por el bienestar de nosotros los estudiantes \\
\hline 24 & Me encuentro satisfecho con los contenidos técnicos y metodológicos de las materias \\
\hline 23 & La biblioteca ofrece recursos bibliográficos que permiten cumplir con mis actividades académicas \\
\hline 27 & Cuando estoy dentro de las instalaciones de la Institución me encuentro seguro \\
\hline 38 & La mayoría de mis profesores me trata con respeto y profesionalismo \\
\hline 43 & $\begin{array}{l}\text { La programación de clases (horarios, secciones y ofertas de las mismas) me permite completar mis } \\
\text { estudios en un tiempo razonable }\end{array}$ \\
\hline 37 & $\begin{array}{l}\text { Las evaluaciones (notas de exámenes, trabajos escritos u orales, y otras) que me realizan los profesores } \\
\text { miden el nivel de la labor académica que he realizado }\end{array}$ \\
\hline 41 & Los laboratorios cuentan con los equipos y materiales necesarios \\
\hline 40 & El personal administrativo se preocupa por darme una excelente atención \\
\hline 42 & $\begin{array}{l}\text { La orientación que me ofrecen los profesores sobres las materias electivas que debo elegir, me ayuda } \\
\text { al fortalecimiento de mis metas académicas y profesionales y cumplirlas }\end{array}$ \\
\hline 48 & El proceso de inscripción es rápido, sencillo y cómodo \\
\hline 47 & Las aulas de clase son cómodas y adecuadas para concentrarme en mis estudios \\
\hline 30 & La Institución cuenta con suficientes estacionamientos para los estudiantes \\
\hline 32 & En los cafetines de la Institución se encuentra comida balanceada y a un precio accesible \\
\hline 45 & $\begin{array}{l}\text { La Institución me brinda experiencias prácticas, fuera del aula, que me capacitan para el mundo del } \\
\text { trabajo }\end{array}$ \\
\hline
\end{tabular}

Validez de contenido: evalúa la correspondencia de las variables contenidas en la escala con su definición conceptual, en otras palabras, se refiere al grado en que un instrumento refleja un dominio específico de contenido de lo que se mide (Hernández, Fernández y Baptista, 2010). Para ello se considera el juicio investigadores y exportados del tema sobre el instrumento a utilizar (Martin, 2004). El objetivo es asegurar que los ítems de la escala cumplan con aspectos empíricos, con consideraciones teóricas y también prácticas (Hair et al. 2010). La encuesta aplicada es una adaptación de la encuesta del modelo SEUing, realizado considerando la revisión bibliográfica relacionada con la satisfacción estudiantil en educación superior, por lo que puede considerarse que la escala presentada presenta validez de contenido.

La validez de criterio: establece la validez de un instrumento confrontándola con algún criterio externo, siendo este un estándar que permite juzgar el test, mientras más se relacionen los resultados con este criterio la validez de criterio será mayor, si el criterio se fija en el presente se habla de validez recurrente y si esté es fijado en el futuro se habla de validez predictiva (Hernández et al., 2010).

Para validez concurrente, mide el grado de correlación de dos medidas del mismo concepto. Se realizó un análisis basado en las percepciones de los estudiantes, para ello se clasificaron los mismos en dos categorías, (Mejías 2005b; Hair et al. 2010), los que presentan baja percepción y los que presentan alta percepción, para esta se comparó sus puntuaciones con respecto a la mediana siendo los de baja aquellos cuyos valores son inferiores a esta, y los que sus medidas sean superiores a la mediana como los de alta percepción. Luego se determinó si existía diferencias significativas entre ambas categorías relacionándolos con las preguntas Tus sentimientos hacia el servicio que se presta en esta dependencia y En general, la calidad de los servicios que recibes en esta dependencia es, de manera que si se produjeran diferencias entre dichas medias se podría decir que la escala es válida. Este análisis se realizó mediante la prueba no paramétricas $U$ de Mann Whitney (Montgomery 
y Runger, 2011), el resultado obtenido, valor de $p<0.05$, indica que la escala presenta validez concurrente.

La validez predictiva, se realizó un análisis de regresión, (Montgomery y Runger, 2011) entre la variable Los estudiantes de esta escuela están satisfechos con los servicios prestados como variable dependiente $(\mathrm{Y})$ y los promedios de las dimensiones como variables independientes (Xi). El valor del coeficiente de determinación (R2) y las pruebas de adecuación respectivas, fueron significativas al 1\%. Esto infiere que la medición de la calidad de servicio en función de las percepciones presenta una predicción de la satisfacción, esto fue comparado con las investigaciones anteriores (Mejías, 2005a) por lo que se establece que el instrumento posee validez predictiva.

La validez de constructo o de construcción, es considerada la validez más importante desde el punto de vista científico, (Hernández, Fernández y Baptista, 2010), y mide que tan exitosamente un instrumento representa y mide un concepto teórico (Grinell, Williams y Unrau, 2009). Se refiere al grado en que el instrumento refleja la teoría del fenómeno o del concepto que mide, este garantiza que las medidas que resultan de la aplicación del instrumento puedan ser utilizadas y consideradas para medir la situación en estudio (Martin, 2004). En esta investigación, se analizó la validez convergente, la cual indica que si la misma existe, se puede medir la situación a estudiar de diferentes formas y los resultados serán parecidos., esta se realizó con base a las preguntas Tus sentimientos hacia el servicio que se presta en esta dependencia y En general, la calidad de los servicios que recibes en esta dependencia es, mediante la prueba de correlación de Spearman, este obtuvo un coeficiente de 0,668 con un p-valor menor a 0,01 , este valor permite establecer que existe validez convergente.

El resultado de un Análisis de Factores arroja información acerca de un instrumento con una determinada muestra pero no de un constructo (GilMonte y Moreno-Jiménez, 2007). Los resultados de un Análisis de Factores dependen de la naturaleza temática y del contenido de los ítems que conforman el cuestionario. En otras palabras, el Análisis de Factores $\mathrm{F}$ no resuelve la naturaleza de un constructo sino de los elementos contenidos en el instrumento, sirve para saber si con ese instrumento realmente estamos midiendo lo que queremos medir.

Para Gil-Monte y Moreno-Jiménez (2007), es un grave error querer identificar el resultado de un Análisis de Factores con un constructo, no es correcto ni metodológica ni epistemológicamente Debe ser al revés, la teoría es la que debe optar por alguna de las alternativas válidas que arroja un Análisis de Factores tanto exploratorio como confirmatorio. También este es un grave error para Seaman y Allen, (2012), quienes aseguran que tan solo es una herramienta estadística para dar validez a un conjunto de datos buscando reducir sus variables descriptoras a un número menor de variables subyacentes que remplace a las variables originales, facilitando información válida para enriquecer el constructo.

\subsection{Análisis DOFA}

Para el análisis del problema y sus resultados se ha decidido aplicar un análisis (Debilidades, Oportunidades, Fuerzas y Amenazas), el cual provee de los insumos necesarios al proceso de planeación estratégica, proporcionando la información necesaria para la implantación de acciones y medidas correctivas y la generación de nuevos o mejores proyectos de mejora (Patiño, 2012; Cadena, 2013).

Partiendo del análisis de contenido del Plan de Desarrollo Institucional (PDI) 2013-2017 (Grijalva, 2014) y con la ayuda de expertos mediante el uso de la Técnica Nominal de Grupo y el Método Delphi, se plantean las fortalezas, debilidades, oportunidades y amenazas que inciden en la satisfacción estudiantil universitaria en la Universidad de Sonora.

\section{El Medio Ambiente Externo: Oportunidades y Amenazas}

Oportunidades para Unisón. Las oportunidades se generan en un ambiente externo, donde la institución no tiene un control directo de las variables. Son eventos que por su relación directa o indirecta pueden afectar de manera positiva el desempeño de la labor académica y administrativa, y por ende, la satisfacción estudiantil.

En este sentido, el PDI contribuye al análisis de las oportunidades guiando el quehacer educativo, da lineamientos sobre las prioridades y en la aplicación de los presupuestos que derivan en planes, programas, proyectos, actividades y metas. Determina cuanto apoyo recibirá del gobierno, el alcance de las actividades, ofrece la oportunidad de diseñar estrategias claras que permitan a la institución alcanzar sus objetivos. Esto lleva a considerar el análisis de la vinculación de la Unisón con los sectores productivos; a revisar aquellos órganos internos y externos que proporcionan recursos a la institución 
Revisando las oportunidades se tendrá una ventana clara de lo que el exterior puede proporcionar con una adecuada selección de estrategias para su aprovechamiento. Así, por ejemplo, la Escuela de Ingeniería Industrial tiene un entorno referido a las necesidades de la industria en el estado (perfiles de puestos, técnicas de manufactura, práctica profesional, avances en la materia, etcétera), regulando la formación de sus egresados y los servicios que proporciona, teniendo capacidad de respuesta para aprovechar las mejoras que en esta materia se den, acumulando para sí las experiencias ajenas que el entorno ofrece.

Amenazas sobre Unisón. Al igual que las oportunidades, las amenazas se encuentran en el entorno de la Institución y de manera directa o indirecta afectan negativamente el quehacer institucional, indicando que se deben tomar las previsiones necesarias para que las amenazas no interrumpan el quehacer universitario ni demeriten su función.

Al igual que las oportunidades, al analizar las amenazas, se deben considerar los factores económicos, los factores políticos y sociales, los productos y la tecnología, los factores demográficos, la competencia y los mercados, entre otros. La situación económica y política del país, por ejemplo, propicia el incremento de la competencia, cada día se generan nuevas escuelas de educación superior, y cada día las demás instituciones educativas, públicas y privadas, se superan en calidad educativa y eficiencia administrativa. La Unisón goza de buena imagen en materia educativa, pero al no considerar esas amenazas, estas se podrían convertir en las causas directas de un descenso fuerte en la captación de alumnos. El análisis de las políticas públicas, del comportamiento del entorno universitario, debe contribuir a definir lo que se debe hacer para impedir que dichas amenazas afecten el desempeño de la institución y sobre todo le impidan lograr los nuevos retos en materia educativa y de satisfacción estudiantil.

\section{El Medio Ambiente Interno: Fortalezas y Debilidades}

Las fortalezas y debilidades internas varían considerablemente para diferentes instituciones; sin embargo, pueden muy bien ser categorizadas en administración y organización, operaciones, finanzas y otros factores específicos para la institución.

Fortalezas de Unisón. Son la parte positiva de la institución de carácter interno, es decir, aquellos productos o servicios que de manera directa se tiene el control de realizar y que reflejan una ventaja ante las demás Instituciones de Educación Superior (IES), producto del esfuerzo y la acertada toma de decisiones.

Las fortalezas se detectan a través de los resultados, por ejemplo, el prestigio de la institución deriva de la calidad académica de sus egresados, de la ocupación de los mejores empleos o la contribución de los egresados en el campo de la investigación y el desarrollo de la tecnología, aun cuando se pueden tener productos intermedios que dan un sello especial a la institución como podría ser el caso de una excelente vinculación que les permite a los egresados tener un nivel mejor de vida y académico. También lo son las modernas instalaciones con las que se cuenta.

Debilidades de Unisón. Es el caso contrario de las fortalezas, las debilidades afectan en forma negativa y directa el desempeño de la institución, derivándose en malos productos o servicios. Las debilidades se podrían atacar con acciones de corto plazo a efecto de eliminarlas y transformarlas en fortalezas; por ejemplo, la deserción escolar es un problema con muchas aristas, tales como la situación económica familiar (amenaza), los malos sistemas de orientación vocacional (debilidad), el poco interés de los maestros en los problemas de los alumnos (debilidad), etcétera. Hay que determinar claramente cuál es la interrelación que existe entre cada uno de los factores FODA $y$, en consecuencia, hacer precisamente acciones estratégicas que permitan que con un movimiento se corrijan dos o más debilidades o se amortigüen dos o más amenazas.

Existen debilidades que con el transcurso del tiempo se pueden convertir en fortalezas como es el caso del Estudios por videoconferencia, que se encuentra en proceso de arranque, pero una vez consolidado pasará a ser una fortaleza. Así pues, las debilidades deberán de ser señaladas con todas sus letras, es decir, no se deben ocultar por intereses diferentes que no sean el proponer y mejorar las políticas y los procesos educativos del instituto para elevar la satisfacción estudiantil universitaria.

\section{Estrategias para elevar la Satisfacción estudiantil Universitaria}

\section{MAXI-MAXI (FORTALEZAS Y OPORTUNIDADES):}

1. Consolidar la oferta educativa en todos sus niveles, el uso de nuevos modelos educativos y metodologías de enseñanza que impulsen la excelencia y la calidad para refrendar las certificaciones. 
2. Fortalecer la presencia de Unisón en aquellas ciudades con bajos índices de cobertura, a través de la formulación de políticas que fomenten la educación a distancia y virtual, especialmente en el área de las ciencias $e$ ingeniería.

3. Dar seguimiento a programas de mejoramiento del profesorado, a efecto de que la institución cuente con una plantilla mejor preparada y con perfil deseable, que realice estudios de posgrado y de actualización.

4. Analizar y actualizar los criterios de evaluación y los indicadores de monitoreo de la función docencia, a efecto de cumplir con los estándares establecidos y que sean susceptibles de ser comparados con los referentes internacionales.

5. Cuidar las modernas instalaciones con que se cuenta y facilitar la estancia estudiantil en ellas y su uso.

\section{MAXI-MINI (FORTALEZAS Y AMENAZAS):}

1. Ofrecer una mayor oferta educativa en las diferentes modalidades, puesto que se encuentra en el $1^{\circ}$ lugar estatal en atender a un mayor número de estudiantes (con un $92 \%$ de la planta docente con estudios de maestría, 25\% con doctorado, pasando dentro de 3 años al $50 \%$ por el grupo académico que se encuentra estudiando doctorado); de la misma manera implementar una metodología, valiéndose de los avances tecnológicos, para el seguimiento de los egresados donde la institución ocupa también el $1^{\circ}$ lugar.

2. Implementar programas de mantenimiento y modernización de la infraestructura en los planteles, consolidar la difusión de contenidos en lo referente al proceso de enseñanzaaprendizaje por medio de publicaciones electrónicas manteniendo así la tendencia positiva en la inscripción de estudiantes.

3. Vincular la formación profesional al mundo del trabajo, elevando la calidad de los programas académicos con lo que se reduciría considerablemente el desempleo y subempleo, puesto que se realizarían evaluaciones diagnósticas confiables y se homogenizaría la calidad de los servicios educativos impartidos.

4. Consolidar, fortalecer y reestructurar los Programas Institucionales, mediante un ejercicio de análisis, revisión minuciosa y detallada de las capacidades de la institución.

\section{MINI-MAXI (DEBILIDADES Y OPORTUNIDADES):}

1. Instrumentar mecanismos que permitan elevar la eficiencia terminal, en especial la orientación vocacional, y se rebase el promedio nacional.

2. Diseñar medidas de retención de alumnos que permitan abatir los índices de deserción en la institución

3. Incrementar la oferta de actualización del personal docente cumpliendo con los criterios de calidad que marcan los organismos como CONACYT.

4. Impulsar que un mayor número de profesores curse estudios de doctorado, a efecto de contar con una plantilla mejor preparada.

5. Revisar la congruencia de los planes y programas de estudio con las demandas y necesidades del estado en términos productivos y laborales.

6. Tener muy en cuenta al personal docente vigente respecto a su edad y al lapso en que se jubilarán, con el propósito de fortalecer la formación de profesores investigadores jóvenes.

\section{MINI-MINI (DEBILIDADES Y AMENAZAS):}

1. Realizar el seguimiento de egresados para mantener vínculos con profesionistas integrados al mundo laboral, a fin de que estas relaciones puedan servir a la institución como herramienta de retroalimentación, para diseñar nuevos y mejores planes y programas apegados al mercado laboral actual para darle oportunidades de trabajo a nuevos egresados.

2. Fortalecer la matrícula en aquellas áreas del conocimiento que presentan mayor demanda en el campo laboral, de acuerdo a la capacidad actual (infraestructura) para una atención educativa de calidad en apego a los nuevos modelos educativos y a los estándares establecidos a nivel internacional.

3. Diseñar programas de formación y actualización del personal académico que permitan impartir educación de calidad y a la altura de Instituciones de prestigio mundial con remuneraciones dignas y acordes con el trabajo académico que realizan, además de promover la incorporación de nuevos esquemas de promoción y de estímulos al desempeño del personal docente.

4. Elaborar un modelo educativo acorde a las características propias de la institución y a las necesidades del estado y del mundo globalizado, que permita contar con planes y programas con contenidos concretos y provistos de referencias comprensibles, apoyándose en 
las nuevas tecnologías de la información y en la moderna infraestructura con la que se cuenta en laboratorios, talleres, centros de cómputo y bibliotecas, acorde con la experimentación, el contacto directo con los problemas, la formación laboral, la práctica profesional y la investigación.

5. Promover la orientación vocacional y de apoyo económico (becas) para incrementar la eficiencia terminal y evitar la deserción escolar, posibilitando a los estudiantes a concluir sus estudios y alcanzar la acreditación y certificación de los mismos a través de cualquiera de las opciones de titulación vigentes.

\section{CONCLUSIONES}

El modelo de Satisfacción Estudiantil Universitaria de cuatro dimensiones y cincuenta y dos variables, fue aplicado a una muestra de 100 estudiantes de la Universidad de Sonora, Unidad Caborca en la carrera de Ingeniería Industrial. Al aplicar el análisis factorial se obtuvo un modelo de 20 variables que se conglomeran en 4 dimensiones; cada una de estas dimensiones fue renombrada con base en las variables que contiene cada una de ellas: Enseñanza, organización académica, vida universitaria, infraestructura y servicios universitarios; la varianza total explicada por este modelo fue de $67 \%$, muy similar al modelo de seis dimensiones que tuvo una varianza explicada de $67.576 \%$ y aplicados por Martínez y Mejías, (2009) y también por Candelas et al. (2013).

Esta información sirvió para desarrollar propuestas estratégicas a través de un DOFA para mejorar la satisfacción del estudiante en la institución. Si se toman en cuenta estas estrategias para elevar la satisfacción del alumno, la universidad tendrá el reconocimiento como una institución enfocada en el estudiante.

Se logró una alta consistencia interna con valores de Alfa de Cronbach para las dimensiones $\left(\alpha_{1}=0,894\right.$; $\left.\alpha_{2}=0,869 ; \alpha_{3}=0,822 ; \alpha_{4}=0,745\right)$, esto nos dice que el instrumento si mide lo que pretende medir.

En lo referente a las pruebas estadísticas realizadas, estas confirman que la escala SEU presenta validez de criterio, tanto como concurrente como predictiva; así mismo, presenta validez de constructo tanto convergente como discriminante. La validez de contenido se evidencia con la revisión de literatura realizada y la consulta de expertos.

Con base en estos resultados se concluye que el instrumento aplicado SEU (Satisfacción Estudiantil Universitaria) tiene la habilidad de que se puede aplicar en otros entornos universitarios tanto nacionales como internacionales, como ya se ha hecho en México, Venezuela y Chile (Marínez y Mejias, 2009; Torres y Sepúlveda, 2009; Candelas et al. 2013).

Este tipo de trabajo contribuye de una manera significativa al aporte de medir la satisfacción estudiantil en el ámbito mexicano, ya que el sustento esta en esta metodología

La medición de la satisfacción estudiantil en educación superior representa una oportunidad para identificar prioridades de mejora en la gestión en la Universidad de Sonora.

Los resultados reportados evidencian la validez y fiabilidad del instrumento usado.

EnelAnálisis DOFArealizado, se han consideradolos factores económicos, políticos, sociales y culturales que representan las influencias del ámbito externo en la institución y que inciden sobre su quehacer interno, ya que potencialmente pueden favorecer o poner en riesgo el cumplimiento de la Misión institucional. La previsión de esas oportunidades y amenazas posibilita la construcción de escenarios anticipados que permitan reorientar el rumbo de la institución (IPN, 2002).

Las fortalezas y debilidades corresponden al ámbito interno de la institución, por lo que durante el proceso de planeación estratégica, se debe realizar el análisis de cuáles son esas fortalezas con las que se cuenta y cuáles las debilidades que obstaculizan el cumplimiento de sus objetivos estratégicos.

Entre algunas características de este tipo de análisis se encuentra las siguientes ventajas:

- Facilitan el análisis del quehacer institucional que por atribución debe cumplir la institución en apego a su marco jurídico y a los compromisos establecidos en las políticas públicas.

- Facilitan la realización de un diagnóstico para la construcción de estrategias que permitan reorientar el rumbo institucional, al identificar la posición actual y la capacidad de respuesta de nuestra institución.

- Permiten identificar la congruencia entre la asignación del gasto público y su quehacer institucional.

De esta forma, el proceso de planeación estratégica se considera funcional cuando las debilidades son disminuidas, las fortalezas son incrementadas, el impacto de las amenazas es considerado y atendido puntualmente, y el aprovechamiento de 
las oportunidades es capitalizado en el alcance de los objetivos, la Misión y Visión de la institución.

\section{REFERENCIAS BIBLIOGRÁFICAS}

[1] Aceves, V. (2004). Dirección estratégica. México: McGraw-Hill.

[2] Alves, H. y Raposo, M. (2004). La medición de la satisfacción en la enseñanza universitaria: el ejemplo de la Universidade da Beira Interior. Revista Internacional de Marketing Público y No Lucrativo, 1 (1), 73-88.

[3] Babbie E. (2009). The practice of social research, 10th Edition. Ed. Belmont, CA: Wadsworth Publishing Company.

[4] Blázquez, J.; Chamizo, J.; Cano, E. y Gutiérrez, S. (2013). Calidad de vida universitaria: Identificación de los principales indicadores de satisfacción estudiantil. Revista de Educación, 362, 458-484.

[5] Cadena, J. (2013). Estrategias Propuestas para Reducir el Nivel de Burnout en los Mandos Medios de las MIPYMES del Sector de Alimentos y Bebidas con un Proceso de Transformación, en el Estado de Sonora. Tesis Doctoral. Centro Interdisciplinario de Posgrado. Universidad Popular Autónoma del Estado de Puebla (UPAEP). Puebla, México. Septiembre de 2013.

[6] Candelas, C; Gurruchaga, M; Mejías, A; y Flores, L. (2013). Medición de la satisfacción estudiantil universitaria: un estudio de caso en una institución mexicana. Iberoamerican Journal of Industrial Engineering. 5(9). 261-274.

[7] De la Fuente, H.; Marzo, M. y Reyes, M. (2010). Análisis de la satisfacción universitaria en la Facultad de Ingeniería de la Universidad de Talca. Ingeniare. Revista chilena de ingeniería, 18 (3), 350-363. ISSN 0718-3305.

[8] De Vicente y Oliva, M. y Manera, J. (2003). El análisis factorial y por componentes principales. En Lévy, J. y Varela, J. (Comp.), Análisis Multivariable para las Ciencias Sociales (pp. 44-89). Madrid, España: Pearson Educación.

[9] Evans, J. y Lindsay, W. (2008). Administración y control de la calidad, 7a edición. México: Cengage Learning Latin America.

[10] Garmendia, M. (2007). Análisis factorial: una aplicación en el cuestionario de salud general de Goldberg, versión de 12 preguntas. Revista de Salud Pública de Chile. 11(2). 57-65.
[11] Garrido, S. (2004). Dirección Estratégica. México: McGraw-Hill.

[12] Gento, S. y Vivas, M. (2003). El SEUE: un instrumento para conocer la satisfacción de los estudiantes universitarios con su educación. Acción Pedagógica, 12 (2), 16-27.

[12] Gil-Monte, P. y Moreno-Jiménez, B. (2007). El síndrome de quemarse por el trabajo (Burnout). Grupos Profesionales de Riesgo. Madrid: Ed. Ediciones Pirámide.

[13] González, C.; López, L. y Montenegro, H. (2012). Análisis de confiabilidad y de validez del instrumento Course Experience Questionnaire (CEQ). Educación y Educadores. 15(1). 63-78. Disponible en: http://www.redalyc.org/articulo. oa?id=83424040005

[14] Grijalva, H. (2014). Plan de Desarrollo Institucional PDI 2013-2017. Universidad de Sonora. Talleres Gráficos de la Universidad de Sonora. Enero de 2014. Consultado en http:// www.uson.mx/institucional/pdi2013-2017.pdf

[15] Hair, J., Anderson, R., Tatham, R. y Black, W. (2010). Análisis Multivariante. 5ta. Ed. Ed. Pearson Prentice Hall Madrid 2010.

[16] IPN (2002). Metodología para el Análisis FODA. Dirección de Planeación y Organización. Instituto Politécnico Nacional (IPN). México D.F. Marzo de 2002.

[17] Martínez, D. y Mejías, A. (2009). Desarrollo de un Instrumento para Medir la Satisfacción Estudiantil en Educación Superior. Docencia Universitaria, Vol. X, No. 2, 29-47.

[18] Pardo, A. y Ruiz, M. (2002). SPSS 11. Guía para el análisis de datos. Madrid: McGraw-Hill.

[19] Patiño, H. (2012). Conferencia: "Tendencias en el Mundo de la Psicología Organizacional"

[20] XX Congreso Mexicano de Psicología y III Congreso Iberoamericano de Psicología y Salud. Sociedad Mexicana de Psicología, A.C. (SMP). 17 de octubre de 2012.

[21] Peña, D. (2002). Análisis de datos multivariantes. Madrid: McGraw-Hill.

[22] Pérez, C. (2004). Técnicas de Análisi Multivariante de datos.Aplicaciones con SPSS ${ }^{8}$. Madrid: Pearson Education.

[23] Prat, R. y Doval, E. (2003). Construcción y Análisis Estadístico de Escalas. En Lévy, J. y Varela, J. (Comp.), Análisis Multivariable para las Ciencias Sociales (pp. 44-89). Madrid, España: Pearson Educación. 
[24] Seaman, J. y Allen, I. (2012). Statistics Roundtable. Words of Caution. Can reducing variables in factor analysis harm your objectives? Quality Progress,(7), 48-50. July 2012. Recuperado de http://asq.org/qualityprogress/2012/07/statistics-roundtable/wordsof-caution.html y consultado en octubre de 2012.

[25] Streiner, D. y Norman, G. (2008). Health Measurement Scales: A practical guide to their development and use. $4^{\text {th }}$ Edition. Oxford University Press, USA, 2008. ISBN :0199231885, 9780199231881.

[26] Torres, C; y Sepúlveda E. (2009). Aplicación de análisis de factores para identificar dimensiones de calidad en estudiantes de Ingeniería Industrial. Revista Gestão Industrial. 5(4). 205-22.

[27] Vergara, J. y Quesada, V. (2011). Análisis de la calidad en el servicio y satisfacción de los estudiantes de Ciencias Económicas de la Universidad de Cartagena mediante un modelo de ecuaciones estructurales. Revista Electrónica de Investigación Educativa, 13(1), 108-122. Consultado el 26-01-2014 en: http://redie.uabc.mx/vol13no1/contenidovergaraquesada.html

[28] Wiersma, W. y Jurs, S. (2008). Research Methods in Education: An Introduction. $9^{\text {th }}$ Edition . Ed. Pearson. 